\title{
THE BOX PRODUCT OF COUNTABLY MANY COPIES OF THE RATIONALS IS CONSISTENTLY PARACOMPACT
}

\author{
L. BRIAN LAWRENCE
}

\begin{abstract}
By proving the theorem stated in the title, we show that local compactness in the factor spaces is not necessary for paracompactness in the box product.
\end{abstract}

\section{Introduction and theorems.}

History. Over twenty years ago, A. H. Stone first raised the issue of normality and paracompactness in box products ([Kn]-1964). The problem remains in large part unsolved. Suppose $\Lambda$ is a separable metric space. Is $\square^{\omega}(\Lambda)$ paracompact? ${ }^{1}$ The answer is yes if $\Lambda$ is locally compact and the Continuum Hypothesis holds (M. E. Rudin, 1972-[Ru]); but if we take $\Lambda$ to be the irrationals, then $\square^{\omega}(\Lambda)$ is

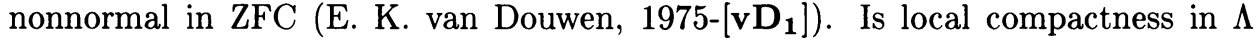
a necessary condition for paracompactness in $\square^{\omega}(\Lambda)$ ? Our answer is no in the presence of either of two combinatorial statements each of which is known to be consistent with ZFC. (In addition to the papers cited in the preceding paragraph, see $\left[\mathbf{K u}, \mathbf{M}, \mathbf{R o}_{\mathbf{1}}, \mathbf{R o}_{\mathbf{2}}, \mathbf{v D}_{\mathbf{2}}, \mathbf{W}_{\mathbf{1}}\right.$ and $\left.\mathbf{W}_{\mathbf{2}}\right]$ for the main results on normality and paracompactness in $\square^{\omega}(\Lambda)$.)

Cardinal numbers. For $\varepsilon, \eta \in{ }^{\omega} \omega$, define $\varepsilon \leq^{*} \eta$ if $(\exists n \in \omega)(\forall m \geq n)\left(\varepsilon_{m} \leq \eta_{m}\right)$. With respect to $\leq^{*}$, let $b$ be the minimal cardinality of an unbounded family and let $d$ be the minimal cardinality of a dominant (cofinal) family. These two cardinal numbers have received much attention in recent years. First note that in ZFC: $\omega_{1} \leq b \leq d \leq c$ ( $c$ is the cardinality of the continuum of real numbers); there is a $\leq^{*}$ well-ordered unbounded family of order type $b$, so $b$ is regular; $b \leq$ cof $d$ and the value of $d$ is the same for eventual domination as it is for strict domination where we require $\varepsilon_{m} \leq \eta_{m}$ on all indices $m$.

It is consistent with ZFC to simultaneously change any of the above relations to either strict equality or strict inequality. In particular, both the Continuum Hypothesis and Martin's Axiom imply $b=d=c$. The equality $b=d$ holds iff there

Received by the editors February 18, 1987 and, in revised form, August 13, 1987. Results of this paper were announced at the annual meeting of the American Mathematical Society in January, 1987.

1980 Mathematics Subject Classification (1985 Revision). Primary 54D18; Secondary 54A35, 54B10, 54B20.

Key words and phrases. Paracompactness, box product, continuum hypothesis, Martin's axiom.

The author thanks the referee for many helpful comments on the original version of this paper.

${ }^{1} \square^{\omega}(\Lambda)$ is the box product of $\omega$-many copies of $\Lambda$ : the point-set is a cartesian product over $\omega$ where each factor is $\Lambda$, and a typical base element is a cartesian product where each factor is a proper open set in $\Lambda$.

The author gratefully acknowledges support received from the Howard University Faculty Research Support Grant Program. 
is a well-ordered dominant family (scale). More generally, S. H. Hechler has shown in $[\mathbf{H}]$ that if $M$ is a model of ZFC in which $\alpha$ and $\beta$ are any two regular cardinals with $\omega_{1} \leq \alpha \leq \beta \leq c$, then there is an extension $N$ of $M$ in which $b=\alpha, d=\beta$, and the aleph value of $c$ is unchanged.

If $\Lambda$ is a locally compact metric space, and $b=d$ or $d=c$, then $\square^{\omega}(\Lambda)$ is paracompact (see $\left[\mathbf{W}_{\mathbf{2}}\right]$ for references).

Spaces. Let $Q$ be the rationals with the usual topology, and let $X=\square^{\omega}(Q)$. We remark that both our theorem and proof remain valid if we replace $X$ with any box product of countably many countable metric spaces.

MAIN THEOREM. Suppose $b=d$ or $d=c$. Then $X$ is paracompact; moreover, every open cover of $X$ has an open pairwise disjoint covering refinement (ultraparacompact). (This result is a corollary of the two auxiliary theorems stated below.)

Equivalence classes. For each $s \in X$, let

$$
e(s)=\left\{t \in X:(\exists n \in \omega)(\forall m \geq n)\left(t_{m}=s_{m}\right)\right\} .
$$

Note that the image of $e$ is a partition of $X$. Let $T \subset X$ such that $T$ contains precisely one point from each equivalence class in the image of $e$ (i.e., let $T$ be a transversal).

Order Hypothesis. There is a partial ordering $\preceq$ of $T$ such that $(T, \preceq)$ is a tree (as usual we mean that $\preceq$ is a reflexive, antisymmetric, and transitive relation for which the set of all predecessors of any given point is well-ordered by $\prec)$ where (1) the height has order type $\leq d$ (i.e., every chain has order type $\leq d$ ), and (2) for each $t \in T, \bigcup\{e(s): t \preceq s\}$ is open in $X$.

THEOREM 1. The Order Hypothesis follows from either $b=d$ or $d=c$.

THEOREM 2. The Order Hypothesis implies that $X$ is ultraparacompact.

Problem 1. Does the Order Hypothesis hold in ZFC? If the answer is yes, then the paracompactness of $X$ is also a result in ZFC. At the present time we can only claim that the Order Hypothesis is strictly weaker than either of the combinatorial statements since $b=d$ and $d=c$ are independent of one another.

Problem 2. Can we use the Order Hypothesis corresponding to the box product of countably many locally compact separable metric spaces to prove in ZFC that $\square^{\omega}$ (Reals) is paracompact? For this type of product, we have that $d=c$ implies the Order Hypothesis, but we have no information on the case $b=d$; Theorem 2 remains intact is we replace "ultraparacompact" with "paracompact" in the conclusion.

REMARK. To prove Theorems 1 and 2 we first need to distinguish a special type of limit point in $X$ and indicate its relationship with the cardinal number $d$.

\section{Classification of limit points.}

Basic open sets. Let $B$ be the collection of all subsets $u \subset X$ such that $u$ is a cartesian product where each factor is the rational part of an open interval with irrational endpoints. Note that $B$ is a base for $X$ where each member is both open and closed.

Different kinds of limit points. Suppose $Y \subseteq X$ and $s \in X$ where $s$ is a limit point of $Y$. If $s$ is a limit point of $Y \cap e(s)$, then we will say $S$ is an essential limit 
point of $Y$; otherwise, $s$ is nonessential. (We remark that $s$ is essential iff $s$ is a limit point of a countable subset of $Y$.) So we have partitioned the limit points of $Y$ into two disjoint sets.

Projections. For each $s \in X$ and each $n \in \omega$, let $p_{0, n}(s)=s \mid[0, n)$, and let $p_{n, \omega}(s)=s \|[n, \omega)$ (the vertical bar means restriction). For each $u \in B$ and each $n \in \omega$, let $p_{n}(u)=\left\{s_{n}: s \in u\right\}$, let $p_{0, n}(u)=\left\{p_{0, n}(s): s \in u\right\}$, and let $p_{n, \omega}(u)=$ $\left\{p_{n, \omega}(s): s \in u\right\}$.

Definitions of $\Phi$ AND $\Psi$. Suppose $C \subseteq B$ and $s \in X$. For each $n \in \omega$, define $\Phi_{n}(C, s)=\left\{u \in C: p_{n, \omega}(s) \in p_{n, \omega}(u)\right.$, and $n$ is the least index with this property $\}$. Define

$$
\Psi(C, s)=\left\{u \in C:(\forall n \in \omega)(\exists m \geq n)\left(s_{m} \notin p_{m}(u)\right)\right\} .
$$

So for each $C$ and each $s,\left\{\Phi_{0}(C, s), \Phi_{1}(C, s), \ldots, \Psi(C, s)\right\}$ is a partition of $C$.

LEMMA 1. Suppose $C \subseteq B$ and $s \in X$. Then $s$ is an essential limit point of $\bigcup C$ iff $s$ is a limit point of $\bigcup\left(\bigcup\left\{\Phi_{n}(C, s): n \in \omega\right\}\right)$.

ProOF. Let $Y=\bigcup\left(\bigcup\left\{\Phi_{n}(C, s): n \in \omega\right\}\right)$. Necessity follows from the inclusion $\bigcup C \cap e(s) \subseteq Y$. For sufficiency assume $s \notin \bigcup C$ (the alternative case is immediate) and suppose that $(\forall u \in B$ with $s \in u)(\exists t(u) \in u)(\exists j(u) \in \omega)$ $\left(t(u) \in \bigcup \Phi_{j(u)}(C, s)\right)$. Define $x(u) \in e(s)$ by $p_{0, j(u)}(x(u))=p_{0, j(u)}(t(u))$ and $p_{j(u), \omega}(x(u))=p_{j(u), \omega}(s)$. Then $x(u) \in u$ so $s$ is a limit point of the image of $x$, and $x(u) \in \bigcup \Phi_{j(u)}(C, s)$ so the image of $x$ is contained in $Y$. Thus $s$ is a limit point of $Y \cap e(s)$.

Lemma 2. Suppose $C \subseteq B$ with $|C|<d$. Then every limit point of $\bigcup C$ is essential.

PROOF. Let $s \in X$. If we can show that $s$ is isolated from $\bigcup \Psi(C, s)$, then the result follows by Lemma 1 . For each $u \in \Psi(C, s)$, let $j(u): \omega \rightarrow \omega$ be the strictly increasing sequence whose image is $\left\{m \in \omega: s_{m} \notin p_{m}(u)\right\}$, and choose $k(u): \omega \rightarrow \omega$ so that for each $m$ in the image of $j(u), k(u)_{m}$ is a nonzero integer whose reciprocal is less than the distance between $s_{m}$ and $p_{m}(u)$. By hypothesis we can now choose a sequence $\xi: \omega \rightarrow \omega$ so that $(\forall u \in \Psi(C, s)$ ) ( $\exists$ an infinite set $i(u) \subset \omega)(\forall n \in i(u))\left(\xi_{n}>(k(u) \circ j(u))_{n}\right)$. We can also take $\xi$ to be strictly increasing with all values nonzero. The increasing property of $j(u)$ implies $(\forall n \in \omega)$ $\left(j(u)_{n} \geq n\right)$, so the increasing property of $\xi$ implies $(\forall n \in \omega)\left((\xi \circ j(u))_{n} \geq \xi_{n}\right)$. Therefore $(\forall u \in \Psi(C, s))(\forall n \in i(u))\left((\xi \circ j(u))_{n}>(k(u) \circ j(u))_{n}\right)$. Let $v \in B$ where $s \in v$ and for each $n \in \omega$, the diameter of $p_{n}(v)$ is less than the reciprocal of $\xi_{n}$. Then $(\forall u \in \Psi(C, s))(\forall m \in \operatorname{Image}(j(u) \mid i(u)))\left(p_{m}(u) \cap p_{m}(v)=\varnothing\right)$.

COROllary. Suppose $Y \subset X$ with $|Y|<d$. Then every limit point of $Y$ is essential; and therefore, $\bigcup\{e(y): y \in Y\}$ is closed.

PROOF. Let $s \in X$. For each $y \in Y$ with $e(y) \neq e(s)$, let $u(y) \in B$ such that $y \in u(y)$ and $\left\{n \in \omega: s_{n} \notin p_{n}(u(y))\right\}$ is infinite. By Lemma 2, every limit point of $\bigcup\{u(y): y \in Y$ with $e(y) \neq e(s)\}$ is essential, so $s$ does not belong to the closure and is therefore isolated from $Y-e(s)$.

\section{Proof of Theorem 1.}

Case 1: Suppose $b=d$. Our first step is to construct a special class of open sets: $\left\{\mu_{\alpha}(x): \alpha \in d, x \in X\right\}$. By our combinatorial statement $b=d$, we can 
choose a subcollection $\{\lambda(\alpha): \alpha \in d\} \subset{ }^{\omega} \omega$ which is both dominant (cofinal) and well-ordered with respect to $\leq^{*}$ (a collection of this type is often called a scale). Let $\sigma: Q \rightarrow \omega$ be a 1-1 correspondence. For each $\alpha \in d$ and each $n \in \omega$, let $\gamma(\alpha)_{n}$ be the minimum distance between any two distinct rational numbers belonging to $\left\{\sigma^{-1}(m): m \leq \lambda(\alpha)_{n}+1\right\}$. For each $\alpha \in d$ and each $(x, y) \in X \times X$, let $\delta_{\alpha}(x, y)$ : $\omega \rightarrow$ Nonnegative Reals where the value on $n$ is obtained by first taking the distance between $x_{n}$ and $y_{n}$, and then dividing this number by $\gamma(\alpha)_{n}$. Finally, for each $\alpha \in d$ and each $x \in X$, let $\mu_{\alpha}(x)=\left\{y \in X: \delta_{\alpha}(x, y)\right.$ converges to zero $\}$.

The collection $\left\{\mu_{\alpha}(x): \alpha \in d, x \in X\right\}$ is a base for the open inverse sets (with respect to $e$ ) where the following elementary facts hold for each choice of $\alpha, \beta \in$ $d$ and $x, y \in X$. (1) The set $\mu_{\alpha}(x)$ is both open and closed and is a union of equivalence classes (with respect to $e$ ). (2) If $\alpha \leq \beta$, then $\mu_{\alpha}(x) \supseteq \mu_{\beta}(x)$. (3) Either $\mu_{\alpha}(x)=\mu_{\alpha}(y)$, or $\mu_{\alpha}(x) \cap \mu_{\alpha}(y)=\varnothing$; so $\left\{\mu_{\alpha}(x): x \in X\right\}$ is a pairwise disjoint open cover of $X$. (4) If both $\left\langle\sigma\left(x_{n}\right): n \in \omega\right\rangle \leq^{*} \lambda(\alpha)$ and $\left\langle\sigma\left(y_{n}\right): n \in \omega\right\rangle \leq^{*} \lambda(\alpha)$, and $e(x) \neq e(y)$, then $\mu_{\alpha}(x) \cap \mu_{\alpha}(y)=\varnothing$.

Our second step is to use the dominance of $\{\lambda(\alpha): \alpha \in d\}$ and the observations of the preceding paragraph to construct our partial ordering of $T$. For each $x \in X$, let $\nu(x)=\mu_{\alpha}(x)$ where $\alpha$ is the least ordinal in $d$ such that $\left\langle\sigma\left(x_{n}\right): n \in \omega\right\rangle \leq^{*} \lambda(\alpha)$. Note that for all $x, y \in X$ : either $\nu(x)$ and $\nu(y)$ are disjoint or one is contained in the other; $\nu(x) \supseteq \nu(y)$ iff $y \in \nu(x)$; and $\nu(x)=\nu(y)$ iff $e(x)=e(y)$. For all $t_{1}, t_{2} \in T$, define $t_{1} \preceq t_{2}$ iff $\nu\left(t_{1}\right) \supseteq \nu\left(t_{2}\right)$ (equivalently, $t_{1} \preceq t_{2}$ iff $t_{2} \in \nu\left(t_{1}\right)$ ).

Case 2: Suppose $d=c$. Then we can let $\prec$ be a well-ordering of $T$ in type $d$. Note that for each $t \in T, \bigcup\{e(s): t \preceq s\}$ is open because the complement is closed by the Corollary to Lemma 2 .

REMARK. The existence of a base $\left\{\mu_{\alpha}(x): \alpha \in d, x \in X\right\}$ for the open inverse sets satisfying (1), (2), and (3) in the second paragraph of Case 1 implies that under $b=d$, the quotient space induced by $e$ (the nabla product) is $d$-metrizable. The converse also holds, and the factors can be generalized to nondiscrete metric spaces. This characterization of $b=d$ is due to $\mathrm{S}$. W. Williams in $\left[\mathbf{W}_{\mathbf{1}}\right]$ and van Douwen in $\left[\mathbf{v D}_{\mathbf{2}}\right]$. J. Roitman showed in $\left[\mathbf{R o}_{\mathbf{2}}\right]$ that $d=c$ can be characterized by the existence of a base for the nabla product (with nondiscrete metric factors) where the union of each $<c$ subcollection is closed.

\section{Proof of Theorem 2.}

Hypothesis. Suppose the Order Hypothesis holds, and let $K$ be a given open cover of $X$. We use the lemmas below to construct an appropriate refinement.

Outline of the Proof. The refinement is defined by induction according to the levels of the tree. Let $B^{\prime}=\{u \in B:(\exists r(u) \in T)(u \cap e(r(u)) \neq \varnothing \& u \subseteq \bigcup\{e(t): r(u) \preceq$ $t\})\}$. Note that $B^{\prime}$ is a base for $X$ where for all $u, v \in B^{\prime}, u$ and $v$ are disjoint whenever $r(u)$ and $r(v)$ are incomparable. We define an order-preserving (with respect to $\preceq$ in the domain and set-inclusion in the range) function $C: T \rightarrow$ Power Set $\left(B^{\prime}\right)$ such that for every $t \in T, C_{t}$ is a pairwise disjoint refinement of $K$ which covers $\bigcup\{e(s): s \preceq t\}$ (we also require that each member of $C_{t}$ intersect this union, and that $C_{s}=C_{t}$ if $s$ and $t$ have the same set of predecessors). Then the union of the image of $C$ is an open pairwise disjoint covering refinement of $K$.

In defining $C$ by induction the problem is to show that the union of each initial segment of a branch of the refinement is closed. Since the cardinality of each initial 
segment is $<d$, this problem (by Lemma 2 ) is reduced to showing that each union contains all of its essential limit points.

In 3.1 we introduce an auxiliary concept, $\Theta$-closed, and show that if a pairwise disjoint subcollection of $B$ is $\Theta$-closed, then the union contains all of its essential limit points. Thus, $\cup C_{t}$ is topologically closed if $C_{t}$ is $\Theta$-closed. So as part of our induction hypothesis we assume that $C_{t}$ is $\Theta$-closed. We then give a procedure for extending $C_{t}$ to a larger $\Theta$-closed collection which in addition covers $e(t)$. The remaining sections of the paper serve this purpose.

In 3.2 we extend $\preceq$ to a partial ordering of $X$ so that the restriction of $\prec$ to each equivalence class is a well-ordering of a special type. Also, each class is inserted directly after its representative in $T$. For each $u \in B$, a point $l(u) \in u$ is distinguished $(l(u)$ may or may not be in $T)$, and a subcollection $A \subseteq B$ is defined by $A=\{u \in B:(\forall x \in u)(l(u) \preceq x)\}$. Note that $A \subseteq B^{\prime}$. We construct our refinement of $K$ as a subcollection of $A$.

We cover $e(t)$ by induction according to the well-ordering of $e(t)$ given by the restriction of $\prec$. (So we have inductive processes at two different levels: among different classes, and inside individual classes.) The special nature of the wellordering implies the following proposition. Suppose points are covered in order using members of $A$. Then the union of each initial segment is closed in $e(t)$. However, we also need a $\Theta$-closed extension of $C_{t}$.

In 3.3 we introduce a second auxiliary concept: the product of two members of $A$. In reference to a function $h: A \times A \rightarrow \omega+1$, for each $(u, v) \in A \times A$, we define $u v \in A$ by $p_{0, n}(u v)=p_{0, n}(u)$ and $p_{n, \omega}(u v)=p_{n, \omega}(u) \cap p_{n, \omega}(v)$, where $n=h(u, v)$ (if $h(u, v)=\omega$, then $u v=u$ ). We then establish a second proposition. Suppose that for each pair of new sets admitted in the course of covering $e(t)$ the following equation holds: $u v=u$. Then our extension of $C_{t}$ is $\Theta$-closed.

In 3.4 we unify the lemmas and proceed with the induction arguments to complete the proof of Theorem 2 .

3.1. $\Theta$-closed collections.

DEFINITIONS OF $\sigma, \tau$, AND $l$. Let $\sigma: Q \rightarrow \omega$ be a 1-1 correspondence. Let $\tau: X \rightarrow{ }^{\omega} \omega$ defined by $\tau(s)_{n}=\max \left\{\sigma\left(s_{m}\right): m<n\right\}$ if $n>0$, and $\tau(s)_{0}=0$. Let $l: B \rightarrow X$ defined by $l(u)_{n}=\sigma^{-1}(m)$ where $m=\min \left\{\sigma\left(s_{n}\right): s \in u\right\}$. Note that $l(u) \in u$.

Lemma 3. Suppose $C \subset B$ with $C$ pairwise disjoint, $s \in X$, and $n \in \omega$. Then $\Phi_{n}(C, s)$ is infinite iff $\left\{\tau(l(u))_{n}: u \in \Phi_{n}(C, s)\right\}$ is unbounded.

Proof. Sufficiency follows from the fact that $\tau \circ l$ followed by projection is single-valued. For necessity suppose $\Phi_{n}(C, s)$ is infinite. For each $u \in \Phi_{n}(C, s)$, let $t(u) \in u$ defined by $p_{0, n}(t(u))=p_{0, n}(l(u))$ and $p_{n, \omega}(t(u))=p_{n, \omega}(s) . C$ is pairwise disjoint so $t$ is 1-1; in turn, since each value of $t$ agrees with $s$ on $[n, \omega), p_{0, n} \circ t$ is 1-1. The conclusion now follows since for a fixed $n$, an infinite number of distinct $n$-tuples collectively assume an infinite number of integer values.

Definition OF $\Theta$-CLOSED. For each $u \in B$, let $\Theta(u): \omega \rightarrow$ Power Set $(X)$ defined by $\Theta(u)_{n}=\left\{s \in X:\left(u \in \Phi_{n}(B, s)\right) \&\left(\tau(s)_{n} \leq \tau(l(u))_{n}\right)\right\}$. A collection $C \subseteq B$ is $\Theta$-closed means that $(\forall u \in C)(\forall n \in \omega)\left(\Theta(u)_{n} \subseteq \bigcup C\right)$.

Lemma 4. Suppose $C \subseteq B$ with $C$ pairwise disjoint and $\Theta$-closed, $s \in X$, and $n \in \omega$ such that $\Phi_{n}(C, s)$ is infinite. Then $s \in \bigcup C$. 
Proof. By Lemma 3 we can choose $u \in \Phi_{n}(C, s)$ with $\tau(s)_{n} \leq \tau(l(u))_{n}$. So $s \in \Theta(u)_{n}$, which in turn is a subset of $\bigcup C$ by hypothesis.

Lemma 5. Suppose $C \subseteq B$ is pairwise disjoint and $\Theta$-closed. Then $\bigcup C$ contains all of its essential limit points.

Proof. Let $s \in X-\bigcup C$. By the contrapositive of Lemma $4, \Phi_{n}(C, s)$ is finite for each $n \in \omega$, so we can choose $u \in B$ with $s \in u$ such that $(\forall n \in \omega)$ $\left(p_{n}(u) \subset Q-\bigcup\left\{p_{n}(v): v \in \Phi_{n+1}(C, s)\right\}\right)$. Since $\Phi_{0}(C, s)$ is empty, $u$ does not intersect any set belonging to $\bigcup\left\{\Phi_{n}(C, s): n \in \omega\right\}$. By Lemma $1, s$ is not an essential limit point of $\bigcup C$.

\subsection{Well-ordering equivalence classes.}

DEFINITIONS OF $f$ AND $g$. Let $f: X \rightarrow \omega$ by defining $f(s)$ to be the least index $n$ with $p_{n, \omega}(s)=p_{n, \omega}(t)$ where $t \in T$ and $e(s)=e(t)$. Let $g: X \times X \rightarrow \omega+1$ defined by setting $g(s, t)$ equal to the least index $n$ with $p_{n, \omega}(s)=p_{n, \omega}(t)$ if $e(s)=e(t)$, and otherwise, let $g(s, t)=\omega$.

DEFINITION OF $\preceq$ ON $X-T$. Extend $\preceq$ to a partial ordering of $X$ so that for all $x, y \in X$ :

(1) if $s$ and $t$ are distinct elements in $T$ with $x \in e(s)$ and $y \in e(t)$, then $x \prec y$ iff $s \prec t$;

(2) if $e(x)=e(y)$ and $f(x)<f(y)$, then $x \prec y$;

(3) if $e(x)=e(y), f(x)=f(y)$, and $\sigma\left(x_{n-1}\right)<\sigma\left(y_{n-1}\right)$ where $n=g(x, y)$, then $x \prec y$.

Note that (2) and (3) imply that $\prec$ well-orders each equivalence class and places its representative in $T$ in the initial position. Also, for each $x \in X,\{y \in X: x \preceq y\}$ is an open set.

Definitions of $A$ And $\Delta$. Let $A=\{u \in B:(\forall x \in u)(l(u) \preceq x)\}$. Let $\Delta=\{C \subseteq A: C$ is pairwise disjoint; each member of $C$ is a subset of some member of $K ;|C|<d\}$. Note that if $u \in B$ and $s \in u$, then there exists $v \in A$ with $s=l(v)$ and $v \subseteq u$. So in the presence of the Order Hypothesis, $\{v \in A: s=l(v)\}$ is a local base at $s$.

LEMma 6. Suppose $t \in T, s \in e(t)$, and $C \cup D \in \Delta$ such that:

(1) $C \cup D$ covers $\{x \in e(t): x \prec s\}$,

(2) $\bigcup C$ is closed in $X$, and,

(3) $(\forall u \in D)((l(u) \in e(t)) \&(l(u) \prec s))$.

Then $s$ either belongs to $\bigcup(C \cup D)$ or is isolated from $\bigcup(C \cup D)$.

ProOf. Suppose $s \notin \bigcup(C \cup D)$. Partition the predecessors of $s$ in $e(t)$ as follows: place $x$ and $y$ in the same partition set provided that $(\exists n \leq f(s))((g(s, x)=$ $\left.g(s, y)=n) \&\left(x_{n-1}=y_{n-1}\right)\right)$.

Claim 1. The partition defined above is finite. The first step is to separate $x$ and $y$ if $g(s, x) \neq g(s, y)$. Since each of $x$ and $y$ precedes $s, f(s)$ is an upper bound for the image of this restriction of $g$, so the first step yields a finite number of sets. If $g(s, x)=g(s, y)=n$, then the second step is to separate $x$ and $y$ in case $x_{n-1} \neq y_{n-1}$. By the definition of $\preceq$ on $X$, each of $x_{n-1}$ and $y_{n-1}$ belongs to $\left\{q \in Q: \sigma(q)<\sigma\left(s_{n-1}\right)\right.$ or $q=t_{n-1}$ (the second possibility can occur when $n=f(s))\}$. So each set constructed initially is broken into only a finite number of subsets in the second step, thus completing the proof of Claim 1. 
A unique representative for each partition set is distinguished by the property of agreeing with $s$ on all but one coordinate. Let $Z$ be the set of all points of this type (so $Z$ is a particular transversal of the partition). By Claim $1, Z$ is finite. Let $E=\{u \in C \cup D: u$ intersects $Z\}$. Since $Z$ is finite and $C \cup D$ is pairwise disjoint, $E$ is finite. So we can choose $w \in B$ with $s \in w$ such that $(\forall u \in E)(\forall i<f(s))$ $\left(\left(s_{i} \notin p_{i}(u) \rightarrow p_{i}(u) \cap p_{i}(w)=\varnothing\right) \&\left(s_{i} \in p_{i}(u) \rightarrow p_{i}(w) \subseteq p_{i}(u)\right)\right)$. Note that by our starting assumption on $s$, the first condition implies $(\forall u \in E)(u \cap w=\varnothing)$.

Claim 2. $\bigcup D \cap w=\varnothing$. Let $v \in D$. By (3) of the hypothesis, $l(v) \in e(t)$ and $l(v) \prec s$. Let $x \in Z$ where $x$ belongs to the same partition set that contains $l(v)$. By (1) of the hypothesis, we can choose $u \in C \cup D$ with $x \in u$. So $u \in E$. Either $u=v$ or $u \cap v=\varnothing$. In the first case $v \cap w=\varnothing$ by the first condition in the choice of $w$. In the second case, if $m=g\left(x, l(v)\right.$ ), then $p_{0, m}(u) \cap p_{0, m}(v)=\varnothing$ (by $u \cap v=\varnothing$ ) while $p_{0, m}(w) \subseteq p_{0, m}(u)$ (by the second condition in the choice of $w$ and the equality $s_{i}=x_{i}$ for all $\left.i \neq g(s, x)-1\right)$; so again we have that $v \cap w=\varnothing$, thus completing the proof of Claim 2.

By Claim 2,s is isolated from $\bigcup D$; and by (2) of the hypothesis together with our starting assumption, $s$ is isolated from $\bigcup C$.

LEMMA 7. Suppose $t \in T$ and $s, x, y \in e(t)$ with $s \preceq x \preceq y$. Then $g(s, x) \leq$ $g(s, y)$.

Proof. Define $i, j, k \in \omega$ by $i=f(s), j=f(x)$, and $k=f(y)$. By (2) in the definition of $\preceq$ on $X, i \leq j \leq k$; and as a consequence, $g(s, x) \leq j$ and $g(s, y) \leq k$.

Case 1. Suppose $i<j$ or $j<k$. Then $i<k$ which implies $g(s, y)=k$.

Case 2. Suppose $i=j=k$. By (3) in the definition of $\preceq$ on $X$, if $g(s, y)<g(s, x)$, then either $x$ precedes each of $s$ and $y$ in the well-ordering or follows each of $s$ and $y$, and this result contradicts the hypothesis.

Definition of $h$. Define $h: A \times A \rightarrow \omega+1$ as follows. Let $h(u, v)$ be the least index $n$ such that $p_{n, \omega}(l(u)) \in p_{n, \omega}(v)$ and $\tau(l(v))_{n} \leq \tau(l(u))_{n}$ if such an index exists; otherwise, let $h(u, v)=\omega$. Note that by the definition of $l$, if $h(u, v)<\omega$, then $(\forall m \geq h(u, v))\left(\tau(l(v))_{m} \leq \tau(l(u))_{m}\right)$. Also, if $l(u)=l(v)$, then $h(u, v)=0$.

LEMMA 8. Suppose $u, v \in A$, with $e(l(u))=e(l(v))$. If furthermore $l(u) \preceq l(v)$, then $g(l(u), l(v)) \leq h(u, v)$.

ProOF. The case $l(u)=l(v)$ is immediate. Assume $l(u) \prec l(v)$. Let $n=$ $g(l(u), l(v))$. Define $s \in X$ by $s_{m}=l(v)_{m}$ for all $m \neq n-1$, and $s_{n-1}=l(u)_{n-1}$.

Claim. $s \prec l(v)$. Define $i, j, k \in \omega$ by $i=f(s), j=f(l(u))$, and $k=f(l(v))$. Then either $j<k$ forcing $i<k$, or $j=k$ forcing $i=k$. In the first case, $s \prec l(v)$ by (2) in the definition of $\preceq$ on $X$. In the second case, by (3) in the definition of $\preceq$ on $X, \sigma\left(l(u)_{n-1}\right)<\sigma\left(l(v)_{n-1}\right)$, so $s \prec l(v)$ follows from $s_{n-1}=l(u)_{n-1}$.

If $h(u, v)<n$, then $s \in v$, and by the Claim, this contradicts $v \in A$.

LemmA 9. Suppose $u, u_{0}, v, v_{0} \in A$ with $u_{0} \subseteq u, v_{0} \subseteq v, l(u)=l\left(u_{0}\right)$, and $l(v)=l\left(v_{0}\right)$. Then $h(u, v) \leq h\left(u_{0}, v_{0}\right)$.

PROOF. Suppose $n<h(u, v)$. Then either $p_{n, \omega}(l(u)) \notin p_{n, \omega}(v)$, and thus $p_{n, \omega}\left(l\left(u_{0}\right)\right) \notin p_{n, \omega}\left(v_{0}\right)$; or $\tau\left(l\left(v_{0}\right)\right)_{n}=\tau(l(v))_{n}>\tau(l(u))_{n}=\tau\left(l\left(u_{0}\right)\right)_{n}$. In neither case does $n=h\left(u_{0}, v_{0}\right)$. 


\subsection{Method of refinement.}

Definition OF THE PRODUCT. For each $(u, v) \in A \times A$, define the product $u v \in A$ by $p_{0, n}(u v)=p_{0, n}(u)$ and $p_{n, \omega}(u v)=p_{n, \omega}(u) \cap p_{n, \omega}(v)$ where $n=h(u, v)<$ $\omega$; and if $h(u, v)=\omega$, then let $u v=u$. Note that $u v \subseteq u$; and $l(u v)=l(u)$ by the definition of $h$, so $u v \in A$. The equation $u v=u$ holds iff $h(u, v)=\omega$, or, $p_{n, \omega}(u) \subseteq p_{n, \omega}(v)$ where $n=h(u, v)<\omega$. Also, if $l(u)=l(v)$, then $u v=u \cap v$.

LEMMA 10. Suppose $s, t \in X$ with $e(s)=e(t)$ and $s \prec t$, and suppose $u, v \in A$ with $l(u)=s$ and $l(v)=t$. Then $u \cap \overleftarrow{t}=u v \cap \overleftarrow{t}$, where $\overleftarrow{t}=\{x \in e(t): x \prec t\}$.

Proof. Since $u v \subseteq u$, one inclusion is immediate. Let $x \in u \cap \overleftarrow{t}$. By $u \in A$, $s \preceq x$. Let $n=h(u, v)$. By Lemmas 7 and $8, g(s, x) \leq g(s, t) \leq n$, so $p_{n, \omega}(x)=$ $p_{n, \omega}(s) \in p_{n, \omega}(u v)$. This result together with $p_{0, n}(x) \in p_{0, n}(u)=p_{0, n}(u v)$ implies that $x \in u v$.

LEMma 11. Suppose $u \in A$ and $C$ is a nonempty subcollection of $A$ such that either:

(1) $C$ is pairwise disjoint, or,

(2) $(\forall v \in C)((e(l(u))=e(l(v))) \&(l(u) \preceq l(v)))$, and, the restriction of $l$ to $C$ is $1-1$.

Let $w=\bigcap\{u v: v \in C\}$. Then $w \in A, w \subseteq u$, and $l(w)=l(u)$.

PROOF. We need only show that $w$ is open. The other properties then follow immediately from $u \in A, u v \subseteq u, l(u v)=l(u)$. The following two facts imply that $w$ is open: $(\forall n \in \omega)\left(p_{n}(w)=\bigcap\left\{p_{n}(v): v=u\right.\right.$, or $v \in C$ with $\left.\left.h(u, v) \leq n\right\}\right)$; $(\forall n \in \omega)(\{v \in C: h(u, v)=n\}$ is finite). The second fact follows from the proof of Lemma 3 if we take the first hypothesis, and from Lemma 8 and the 1-1 property of $l \mid C$ if we take the second hypothesis.

Definition of $R$. Suppose $C \subseteq A$ such that $(\forall u, v \in C)(e(l(u))=e(l(v)))$, and the restriction of $l$ to $C$ is $1-1$. For each $u \in C$, let $u^{\prime}=\bigcap\{u v: v \in C$ with $l(u) \preceq l(v)\}$. By Lemma 11(2), $u^{\prime}$ is open. Let $R(C)=\left\{u^{\prime}: u \in C\right\}$.

LEMMA 12. Suppose $C \subseteq A$ such that:

(1) $(\forall u, v \in C)(e(l(u))=e(l(v)))$,

(2) the restriction of $l$ to $C$ is 1-1,

(3) $(\forall u \in C)(\forall v \in R(\overleftarrow{u}))(u v=u)$, where $\overleftarrow{u}=\{w \in C: l(w) \prec l(u)\}$

Then $(\forall u, v \in R(C))(u v=u)$.

ProOF. Suppose $u, v \in C$ and let $u^{\prime}$ and $v^{\prime}$ be the corresponding members of $R(C)$. We will prove that $u^{\prime} \subseteq u^{\prime} v^{\prime}\left(u^{\prime} v^{\prime} \subseteq u^{\prime}\right.$ follows immediately from the definition of the product). Also by the definition of the product: $u^{\prime} \subseteq u^{\prime} v^{\prime}$ iff $p_{n, \omega}\left(u^{\prime}\right) \subseteq p_{n, \omega}\left(v^{\prime}\right)$, where $n=h\left(u^{\prime}, v^{\prime}\right)$; and in turn, the projections of $v^{\prime}$ are intersections of projections of certain members of $C$. Suppose $w \in C$ with $l(v) \preceq$ $l(w)$. Let $n_{1}=h\left(u^{\prime}, v^{\prime}\right)$, let $n_{2}=h(v, w)$, and let $m=\max \left\{n_{1}, n_{2}\right\}$. We need to show that $p_{m, \omega}\left(u^{\prime}\right) \subseteq p_{m, \omega}(w)$ (we can assume $m<\omega$ since otherwise, $u^{\prime}=u^{\prime} v^{\prime}$ is immediate (if $n_{1}=\omega$ ) or $w$ is irrelevant in the construction of $v^{\prime}\left(\right.$ if $\left.n_{2}=\omega\right)$ ).

Case 1. Suppose $l(u) \preceq l(v)$. By Lemma 11(2), $l\left(u^{\prime}\right)=l(u)$ and $l\left(v^{\prime}\right)=$ $l(v)$, so by Lemma $8, g(l(u), l(v)) \leq n_{1}$. Also by Lemma $8, g(l(v), l(w)) \leq n_{2}$. Thus $g(l(u), l(w)) \leq m$. By $m \geq n_{1}$ and the above application of Lemma 11(2), $\tau(l(v))_{m} \leq \tau(l(u))_{m} ;$ and by $m \geq n_{2}, \tau(l(w))_{m} \leq \tau(l(v))_{m}$. So $h(u, w) \leq m$, and this together with $u^{\prime} \subseteq u w$ implies $p_{m, \omega}\left(u^{\prime}\right) \subseteq p_{m, \omega}(u w) \subseteq p_{m, \omega}(w)$. 
Case 2. Suppose $l(v) \prec l(u) \preceq l(w)$. By Lemma 7, $g(l(u), l(v))=g(l(v), l(u)) \leq$ $g(l(v), l(w))$. As we noted in the preceding paragraph, $g(l(v), l(w)) \leq n_{2}$ by Lemma 8. So $g(l(u), l(w)) \leq m$. The argument can now be completed by the same sequence of ideas used in the conclusion of Case 1.

Case 3. Suppose $l(v) \preceq l(w) \prec l(u)$. Let $z$ be the set in $R(\overleftarrow{u})$ that corresponds to $v$. Then $v^{\prime} \subseteq z \subseteq v w$. By (3) of the hypothesis, $u=u z$. By Lemma 9, $h(u, z) \leq h\left(u^{\prime}, v^{\prime}\right) \leq m$, so we have the following sequence of inclusions:

$$
p_{m, \omega}\left(u^{\prime}\right) \subseteq p_{m, \omega}(u) \subseteq p_{m, \omega}(u z) \subseteq p_{m, \omega}(z) \subseteq p_{m, \omega}(v w) \subseteq p_{m, \omega}(w) .
$$

LEMMA 13. Suppose $C \cup D \in \Delta$ such that:

(1) $C$ is $\Theta$-closed,

(2) $(\forall u \in D)(e(l(u)) \subseteq \bigcup(C \cup D))$,

(3) $(\forall u \in D)(\forall v \in C)(\forall n \in \omega)\left(p_{n, \omega}(u) \cap p_{n, \omega}(v) \neq \varnothing \rightarrow p_{n, \omega}(u) \subseteq p_{n, \omega}(v)\right)$,

(4) $(\forall u, v \in D)(u v=u)$.

Then $C \cup D$ is $\Theta$-closed.

Proof. Let $u \in C \cup D$, let $n \in \omega$, and let $s \in \Theta(u)_{n}$. We need to show that $s \in \bigcup(C \cup D)$.

Case 1. Suppose $u \in C$. Then $s \in \bigcup C$ by (1) of the hypothesis.

Case 2. Suppose $u \in D$. Let $t \in e(l(u))$ be defined by $p_{0, n}(t)=p_{0, n}(s)$ and $p_{n, \omega}(t)=p_{n, \omega}(l(u))$. By (2) of the hypothesis, we can choose $v \in C \cup D$ with $t \in v$.

Claim. $s \in v$. By $t \in v, p_{0, n}(s) \in p_{0, n}(v)$. We will show that $p_{n, \omega}(u) \subseteq p_{n, \omega}(v)$, from which $p_{n, \omega}(s) \in p_{n, \omega}(v)$ follows by the choice of $s$. The above inclusion holds by (3) of the hypothesis if $v \in C$, and by (4) of the hypothesis and $h(u, v) \leq n$ if $v \in D$. The inequality $h(u, v) \leq n$ is a consequence of two facts: $p_{n, \omega}(l(u))=$ $p_{n, \omega}(t) \in p_{n, \omega}(v)$ by the choice of $t$ and $v$, and $\tau(l(v))_{n} \leq \tau(t)_{n}=\tau(s)_{n} \leq \tau(l(u))_{n}$ by the choice of $t$ and $s$.

\subsection{Transfinite induction.}

LeMma 14. Suppose $C$ is a $\Theta$-closed member of $\Delta$ and $t \in T$ such that $C$ covers $e(s)$ for each $s \prec t$. Then there is a collection $D$ such that $C \cup R(D)$ is a $\Theta$-closed member of $\Delta$ that covers $e(t)$.

ProOF. To construct $D$ we will use transfinite induction on $e(t)$ ordered by $\prec$. For the induction hypothesis, suppose $D$ has been partially constructed so that the conditions listed below are satisfied (for each $u \in D$, let $\overleftarrow{u}=\{v \in D: l(v) \prec l(u)\}$ ):

(1) $C \cup R(D) \in \Delta$;

(2) $(\forall u \in D)(l(u)$ is the least element of $e(t)-\bigcup(C \cup R(\overleftarrow{u})))$;

(3) $(\forall u \in D)(\forall v \in R(\overleftarrow{u}))(u v=u)$;

(4) $(\forall u \in D)(\forall v \in C)(\forall n \in \omega)\left(p_{n, \omega}(u) \cap p_{n, \omega}(v) \neq \varnothing \rightarrow p_{n, \omega}(u) \subseteq p_{n, \omega}(v)\right)$. (If we define $\left(4^{\prime}\right)$ to be the same as (4) except with $R(D)$ in place of $D$, then (4) implies $\left(4^{\prime}\right)$.)

First note that by Lemma 12 , the above hypothesis implies $(\forall u, v \in R(D))$ $(u v=u)$; so by Lemma 13, if $C \cup R(D)$ convers $e(t)$, then $C \cup R(D)$ is $\Theta$-closed. Therefore, we need to show that in the case where $e(t)$ is not yet covered, our collection can be extended while preserving each of the conditions of the induction hypothesis.

Claim. Let $x$ be a minimal element of $X$ such that $t \preceq x$ and $(\forall u \in D)(l(u) \prec x)$, and let $y \in e(t)$ with $y \prec x$. Then $y \in \bigcup(C \cup R(D))$. First note that by Lemma 
11(2), $\{l(u): u \in D\}=\{l(u): u \in R(D)\}$. Suppose $y \notin \bigcup C$ and $y$ does not belong to the image of $l$ restricted to $D$. Let $u \in D$ such that $l(u)$ is the least element of $\{l(v):(v \in D) \&(y \prec l(v))\}$. By (2) of the induction hypothesis, we can choose $w \in \overleftarrow{u}$ with $y \in z_{0}=\bigcap\{w v:(v \in D) \&(l(w) \preceq l(v) \prec l(u))\}$. Let $z_{1}=\bigcap\{w v:(v \in D) \&(l(u) \preceq l(v))\}$. By Lemma 10, $y \in z_{1}$. Let $w^{\prime}=z_{0} \cap z_{1}$. Then $y \in w^{\prime} \in R(D)$. This completes the proof of the Claim.

Let $s$ be the least element of $e(t)-\bigcup(C \cup R(D))$. By the Claim, $x \preceq s$. By Lemmas 2 and $5, \cup C$ is closed in $X$. Therefore $t, s$, and $C \cup R(D)$ satisfy the hypothesis of Lemma 6 , so we can choose $w_{1} \in B$ where $s \in w_{1}, w_{1}$ is a subset of some member of $K$, and $w_{1}$ is disjoint from $\bigcup(C \cup R(D))$. Since $\{v \in A: s=$ $l(v)\}$ is a local base at $s$, we can choose $w_{2} \in A$ with $s=l\left(w_{2}\right)$ and $w_{2} \subseteq w_{1}$. By the contrapositive of Lemma $4, \Phi_{n}(C, s)$ is finite for each $n \in \omega$, so we can choose $w_{3} \in A$ with $s \in w_{3}$ and $w_{3} \subseteq w_{2}$ such that $(\forall n \in \omega)\left(\forall v \in \Phi_{n+1}(C, s)\right)$ $\left(p_{n}(v) \cap p_{n}\left(w_{3}\right)=\varnothing\right)$, and $(\forall n \in \omega)\left(\forall v \in \Phi_{n}(C, s)\right)\left(p_{n, \omega}\left(w_{3}\right) \subseteq p_{n, \omega}(v)\right)$. Finally, if $D$ is nonempty, then let $u=\bigcap\left\{w_{3} v: v \in R(D)\right\}$; otherwise, let $u=w_{3}$. By Lemma 11(1), $u \in A, u \subseteq w_{3}$, and $s \in u$. By virtue of Lemma 9 and the intersection that defines $u,(\forall v \in R(D))(u v=u)$. Adjoin $u$ to $D$. Then the extension satisfies each condition of the induction hypothesis.

\section{LEMMA 15. There is an open pairwise disjoint covering refinement of $K$.}

PROOF. By Lemma 14 and transfinite induction, there is an order-preserving (with respect to $\preceq$ in $T$ and set inclusion in $\Delta$ ) function $C: T \rightarrow \Delta$ such that for every $t \in T, C_{t}$ is a $\Theta$-closed member of $\Delta$ that covers $\bigcup\{e(s): s \prec t\}$ (we also require that each member of $C_{t}$ intersect this union, and that $C_{s}=C_{t}$ if $s$ and $t$ have the same set of predecessors). Then the union of the image of $C$ is a refinement of $K$ with the required properties.

\section{REFERENCES}

[vD 1 E. K. van Douwen, The box product of countably many metrizable spaces need not be normal, Fund. Math. 88 (1975), 127-132.

[ $\left.\mathbf{v D}_{\mathbf{2}}\right]$, Covering and separation in box products, Surveys in General Topology, Academic Press, 1980, pp. 55-130.

[H] S. H. Hechler, On the existence of certain cofinal subsets of ${ }^{\omega} \omega$, Proc. Sympos. Pure Math., vol. 10, Part 2, Amer. Math. Soc., Providence, R.I., 1974, pp. 155-174.

[Kn] C. J. Knight, Box topologies, Quart. J. Math. 15 (1964), 41-54.

[Ku] K. Kunen, On paracompactness of box products of compact spaces, Trans. Amer. Math. Soc. 240 (1978), 307-316.

[M] A. W. Miller, On box products, Topology Appl. 14 (1982), 313-317.

[Ro $]$ J. Roitman, Paracompact box products in forcing extensions, Fund. Math. 102 (1978), 219228.

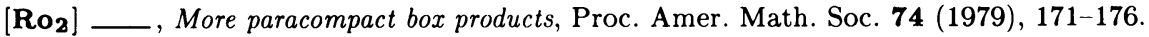

[Ru] M. E. Rudin, The box product of countably many compact metric spaces, General Topology and Appl. 2 (1972), 293-298.

[W1] S. W. Williams, Is $\square^{\omega}(\omega+1)$ paracompact?, Topology Proc. 1 (1976), 141-146.

$\left[\mathbf{W}_{\mathbf{2}}\right]$ _ Box products, Handbook of Set-Theoretic Topology, K. Kunen and J. Vaughan, Eds., Elsevier, 1984, pp. 169-200.

\section{Department of Mathematics, Howard University, Washington, D.C. 20059}

Current address: Department of Mathematics, George Mason University, Fairfax, Virginia 22030 